Pacific Journal of Mathematics

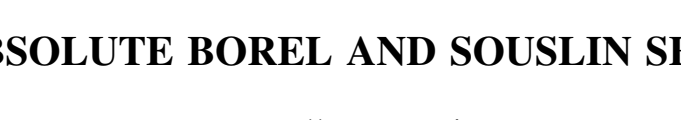




\title{
ABSOLUTE BOREL AND SOUSLIN SETS
}

\author{
ZDENĚK FROLÍK
}

The theory of analytic, Borelian and absolutely Baire spaces is applied to the theory of absolutely Souslin and Borel Sets with respect to the class of all metrizable spaces. Several intrinsic characterizations of absolutely Souslin and Borel sets are given.

The basic idea is that if there is given a class $\mathscr{A}$ of respectable sets in a separable theory, then the corresponding class $\mathscr{C}$ in the associated nonseparable theory consists of all spaces $P$ such that $P=A \cap G$ in $\beta P$ where $A \in \mathscr{A}$ and $G$ is a $G_{\delta}$ set in $\beta P$. If the elements of $\mathscr{A}$ are characterized by existence of a complete structure of certain type, then the elements of $\mathscr{C}$ are characterized by the existence of a complete bi-structure $\langle\alpha, \beta\rangle$ where $\alpha$ is closely related to the structure defining $\mathscr{A}$, and $\beta$ is closely related to the structure characterizing absolute $G_{\delta}$ spaces. This approach to the nonseparable theory is discussed for analytic, Borelian and bi-analytic spaces. The theory is applied to absolutely Borel and Souslin sets in the class of all metrizable spaces.

Call a space $P$ an absolute Borel space (an absolute Souslin space) if $P$ is metrizable, and a Borel (Souslin) set in every metrizable $Q \supset P$. By a Souslin set in a space $Q$ we mean a Souslin set derived from the closed sets of $Q$; a full definition is given below. Since every closed set in a metrizable space is a $G_{j}$, a set $P$ is Borel (Souslin) in $Q \supset P$ if and only if $P$ is Borel (Souslin) in the closure of $P$ in $Q$. A set $X$ in a metrizable space $Q$ is a Borel set (Souslin set) if (and only if) $X$ is Borel (Souslin) in the closure of $X$ in $Q$, or in any Borel set $Y \supset X, Y \subset Q$.

There is a rather extensive and deep theory of separable absolute Borel and Souslin sets. The basic facts from the separable theory were generalized to more general spaces by several authors, and a closed separable theory in the class of all uniformizable spaces is developed in [2], [3], [4]; for a survey see [5] ${ }^{1}$. On the other hand the nonseparable theory is still waiting for new ideas. The best information can be found in A. H. Stone's papers. The results in nonseparable theory strongly resemble the separable theory although more careful proofs are needed. The purpose of this paper is to describe internal characterizations of absolute Borel and Souslin sets

${ }^{1}$ For an up-to-date survey we refer to the author's $A$ survey of descriptive theory of sets and spaces, to appear in the first issue of Czech. Math. J., 1970. All the results needed are discussed in this paper. 
by means, in fact, of the space $\Sigma$ of irrational numbers and topologically complete spaces, and to indicate, by external characterizations meaningful for uniformizable spaces, that the nonseparable theory is more complicated, and the role of compactness nondirect and more delicate. First we recall a few simple results we need.

Theorem 1. If $P$ is a Borel (Souslin) set in an absolute Borel (absolute Souslin) space Q, in particular, if $P$ is an absolute Borel (absolute Souslin) set in a completely metrizable space $Q$, then $P$ is an absolute Borel (absolute Souslin) space.

The proof follows immediately from the following result due to Lavrentiev (cf. [8], p. 135).

If $P_{i} \subset Q_{i}(i=1,2), Q_{i}^{\prime}$ s are completely metrizable, and if $f$ is a homeomorphism of $P_{1}$ onto $P_{2}$, then there exist $G_{\hat{o}}$ sets $G_{i}$ in $Q_{i}$ and a homeomorphism of $G_{1}$ onto $G_{2}$ which is an extension of $f$.

In what follows all the spaces are assumed to be uniformizable and separated. The general terminology and notation is standard.

By a correspondence of $P$ into $Q$ we mean a triple $f: P \rightarrow Q$ such that $f$ is a subset of $P \times Q$; here $P$ and $Q$ are sets (possibly endowed with some structures, e.g., with topologies). The image of a set $X \subset P$ under $f$ is denoted by $f[X]$, if $x \in P$ then $f(x)$ or $f x$ stands for $f[(x)]$ where $(x)$ is a singleton. The letter $\Sigma$ stands for the set of all sequences of natural numbers; with the topology of point-wise convergence $\Sigma$ is known to be the space of irrational numbers. Next, $S$ denotes the set of all finite sequences of natural numbers, $S_{n}$ the set of all $s^{\prime} \mathrm{s}$ in $S$ of length $n$; if $\sigma \in \Sigma$ then $\mathrm{s}<\sigma$ means that $s \in S$ and $s$ is a restriction (called section) of $\sigma$. Also if $t \in S$ then $s<t$ means that $s \in S$ and $s$ is a section of $t$. The section of $\sigma$ of length $n$ is denoted by $\sigma_{n}$. For $s$ in $S$ denote by $\Sigma(s)$ the set of all $\sigma \in \Sigma$ with $s<\sigma$. Clearly $\{\Sigma(s) \mid s \in S\}$ is an open base for the space $\Sigma$ of irrational numbers.

A determining system of subsets of $P$ is a mapping $M$ of $S$ into $\exp P$ such that $M(s) \subset M(t)$ if $t$ is a section of $s$. The symbol $S(M)$ denotes the set

$$
\bigcup\{\cap\{M(s)|s<\sigma| \sigma \in \Sigma\} .
$$

The Souslin sets in a space are of the form $S(F)$, where $F$ is a determining system whose range consists of closed sets. We denote by $M(\sigma)$ the set $\bigcap\{M(s) \mid s<\sigma\}$. Clearly $\boldsymbol{S}(M)=\bigcup\{M(\sigma)\}$. If $N$ is a mapping of $S$ into $\exp P$, and if we set

$$
M s=\bigcap\{N t \mid t<s\},
$$

we get a determining system $M$ with $S(M)=S(N)$. 
Definition. Let $\alpha_{1}$ and $\alpha_{2}$ be two structures on a space $P$ for which the concept of $\alpha_{1}$-Cauchy filter is defined. We say that $\alpha_{1}$ is complete if every $\alpha_{1}$-Cauchy filter has a cluster point; we say $\left\langle\alpha_{1}, \alpha_{2}\right\rangle$ is complete if a filter $\mathscr{C}$ on $P$ has a cluster point provided $\mathscr{C}$ is an $\alpha_{i}$-Cauchy filter, $i=1,2$. The reader is certainly familiar with the definition of Cauchy filters for uniformities or metrics. If $M$ is a determining system then a filter $\mathscr{C l}$ is said to be a Cauchy filter if $M(s) \in \mathscr{C}$ for all $s<\sigma$, for some $\sigma$ in $\Sigma$. If $\alpha_{1}$ is a family $\left\{\mathscr{A}_{a}\right\}$ of collections of sets then a filter $\mathscr{C}$ is a Cauchy filter if $\mathscr{M} \cap \mathscr{C}_{a} \neq$ $\varnothing$ for all $a$.

Recall the following two results which are due to the author.

A space is $G_{\delta}$ in each of its compactifications (i.e., is topologically complete in the sense of E. Čech) if and only if there exists a complete sequence of open coverings of $P$ (see, e.g., [3, §3]).

A space $P$ is analytic (i.e., Souslin in every space) if and only if there exists a complete determining system $M$ on $P$ such that $\boldsymbol{S}(M)=P$ (see, e.g., [2]).

A space $P$ is called analytic if there exists an usco-compact (upper semi-continuous compact-valued) correspondence of $\Sigma$ onto $P$. We refer to [2] for all the properties needed. Recall that $P$ is analytic if and only if $P$ is Souslin in every space $Q \supset P$. We refer to [3] for a development of the theory of complete families of coverings.

1. Souslin spaces. First, an equivalence of four conditions on a uniformizable space will be proved. Then these conditions will be shown to characterize absolute Souslin sets in the class of all metrizable spaces. A tight extension of a space $P$ is a space containing $P$ as a dense subspace.

THEOREM 2. The following conditions on a space $P$ are equivalent:

(a), (a') There exist a sequence $\left\{\mathscr{U}_{n}\right\}$ of open coverings of $P$ and $a$ determining system $M: S \rightarrow \exp P$ with $S(M)=P$ such that $\left\langle\left\{\mathscr{C}_{n}\right\}, M\right\rangle$ is complete (and all $M(s)$ are closed).

(b) $P$ is the intersection of $a G_{o}$ with an analytic set in $\beta P$.

(c) $P$ is the intersection of $a G_{o}$ with an analytic set in some compactification of $P$.

(d) $P$ is the intersection of a $G_{o}$ with a Souslin set in every tight extension of $P$.

Proof. Clearly (a) and (a') are equivalent, and (d) implies (c) as well as (b); it follows from the next Lemma 1 that (a) implies each 
of the conditions (b) through (d). Finally, it follows from Lemma 2 that each of the conditions (b) or (c) implies (a).

Lemma 1. Assume that $Q$ is a tight extension of a space $P$, $\left\{\mathscr{U}_{n}\right\}$ is a sequence of open covers of $P$, and $M: S \rightarrow \exp P$ is a determining system with $S(M)=P$. Let $U_{n}$ be the union of all open subsets $V$ in $Q$ with $V \cap P \in \mathscr{U}_{n} ;$ put $G=\bigcap\left\{U_{n}\right\}$. Define $F: S \rightarrow \exp Q$ by $F(s)=\operatorname{cl} M(s)$ (the closure is taken in. Q), and put $A=\boldsymbol{S}(F)$. If $\alpha=\left\langle\left\{\mathscr{U}_{n}\right\}, M\right\rangle$ is complete (on $P$ ) then $G \cap A=P$, and so $P$ is the intersection of a $G_{\delta}$ with a Souslin set in $Q$.

Proof. Evidently $G \cap A \supset P$ is independent of the completeness of $\alpha$. Let $x \in G \cap A$. There exists an $\sigma$ in $\Sigma$ such that $x \in F(s)$ for all $s<\sigma$. Let $\mathscr{U}$ be the neighborhood system at $x$; consider the filter $\mathscr{V}^{-}=\mathscr{U} \cap P(=E\{U \cap P \mid U \in \mathscr{U}\})$. Because $x \in G, \mathscr{V}^{-} \cap \mathscr{U}_{n} \neq \varnothing$ for each $n$. Then collection $\mathscr{C}$ consisting of all $V \in \mathscr{V}^{-}$and all $M(s), s<\sigma$, is a filter sub-base on $P$, and the smallest filter $\mathscr{M}_{1} \supset \mathscr{M}$ is a $\alpha$-Cauchy filter. Hence there exists a cluster point $y$ of $\mathscr{C}_{1}$ in $P$. But $\mathscr{U}$ converges to $x$ in $Q$, and hence $x=y$ because $Q$ is a separated (that is, Hausdorff) space, and $\mathscr{C}$ dominates $\mathscr{M}_{1}$.

Lemma 2. Assume that $P=G \cap A$ where $G$ is a $G_{o}$ in a compactification $K$ of $P$, and $A$ is an analytic subset of $K$. Choose open $U_{n}$ with $G=\bigcap\left\{U_{n}\right\}$, and an $F: S \rightarrow \exp K$ with $S(F)=A$ such that each $F(s)$ is closed (thence compact). Let $\mathscr{Q}_{n}$ be the collection of all open sets $V$ in $P$ such that the closure of $V$ (taken in $K$ ) is contained in $U_{n}$. Define $M: S \rightarrow \exp P$ by $M(s)=P \cap F(s)$. Then $\alpha=\left\langle\left\{\mathscr{U}_{n}\right\}, M\right\rangle$ is complete.

Proof. Let $\mathscr{C}$ be an $\alpha$-Cauchy filter on $P$. The space $K$ is compact and so $\mathscr{C}$ has a cluster point $x$ in $K$. We shall prove that $x \in P$. There exists a $\sigma$ such that $M(s) \in \mathscr{C}$ for all $s<\sigma$, and so $x \in \cap\{F(s) \mid s<\sigma\} \subset A$. It remains to show that $x \in G$, i.e., $x \in U_{n}$ for all $n$. If $O_{n} \in \mathscr{M} \cap \mathscr{U}_{n}$, then $x \in \operatorname{cl} O_{n} \subset U_{n}$.

REMARK. Assume that $R$ is the closure of $P$ in a space $Q$, and assume that $P=G \cap A$ with $G$ and $G_{\delta}$ in $R$ and $A$ a Souslin (analytic) set in $R$. Then $P=G^{\prime} \cap A$ where $G^{\prime}$ is a $G_{\delta}$ in $Q$, and $A$ is Souslin (analytic) in $Q$. Indeed, choose a $G_{\delta} G^{\prime}$ in $Q$ such that $G^{\prime} \cap R=G$. Then clearly $P=G \cap A$. It follows that the conditions in Theorem 2 are equivalent to condition $c$ with "some compactification of $P$ " replaced by a compact space that contains $P$, and also to condition $c$ with tight delected. 
THEOREM 3. Each of the conditions (a) through (d) in Theorem 2 is necessary and sufficient for a metrizable space $P$ to be an absolute Souslin space.

Proof. By Theorem 2 each of the equivalent conditions (a) through (d) is sufficient. Conversely, if $P$ is an absolute Souslin space then condition (a) is fulfilled by the next lemma (take a completion $Q$ of $P$ for some metric; $\mathscr{V}_{n}^{n}$ is, e.g., the collection of all open sets of diameter less than $1 / n$.

LEMMA 3. Let $\left\{\mathscr{Y}_{n}^{-}\right\}$be a complete sequence of open coverings of a space $Q$ and let $P=\boldsymbol{S}(F)$ where $F$ is a determining system consisting of closed sets of $Q$. Put $\mathscr{C}_{n}=P \cap\left[\mathscr{Y}_{n}\right], M=P \cap[F]$ (i.e., $M(s)=P \cap F(s))$. Then $\left\langle\left\{\mathscr{U}_{n}\right\}, M\right\rangle$ is complete.

Proof. Evident.

Corollary. The following condition on $P$ is equivalent to each of conditions (a) through (d) in Theorem 2:

(e) $P$ is the intersection of $G_{\delta}$ with a Souslin set in some topologically complete (in the sense of E. Čech) space.

Theorem 4. Every absolute Souslin space is the intersection of $a G_{\delta}$ with a Souslin set derived from the zero sets in some compactification of $P$.

Proof. Let $K$ be the Stone-Čech compactification of a completion $Q$ of $P$. By Čech's theorem $Q$ is a $G_{o}$ in $K$. Let $P=\boldsymbol{S}(F)$ where $F$ is a determining system consisting of closed sets in $Q$, thence zero sets in $Q$ because $Q$ is metrizable. For each $s$ choose any zero set $Z(s)$ in $K$ with $Q \cap Z(s)=F(s)$. Put $A=S(Z)$. Clearly $Q \cap S(Z)=$ $S(F)=P$.

REMARK. The proof works for any compactification $K$ of any completion $Q$ of $P$ with the following property: each zero set in $Q$ is a trace of a zero set in $K$. It is clear that every absolute Souslin space $P$ is the intersection of a $G_{o}$ with a Souslin set derived from zero sets in $\beta P$ (because $\beta P$ maps onto any compactification of $P$ ), this is not true for every compactifications, and I don't know whether it is true for compactification with "enough" zero sets.

By Theorem 1 in [4] if $A$ is an analytic subspace of a space $P$ and $X$ is a Souslin set disjoint from $A$, then $A \subset B \subset P-X$ for some Baire set in $P$. I believe that a similar statement for absolute 
Souslin $A$ is false. It might be interesting to describe reasonably the spaces with this property in the class of metrizable $P$ 's. More precisely, call a space $A$ a Lusin space if $A$ is metrizable and the following condition is fulfilled: if $P$ is metrizable, $A \subset P$, and if $X$ is a Souslin set in $P$ disjoint from $A$, then $A \subset B \subset P-X$ for some Baire set in $P$. By a Baire set in $P$ we mean an element of the smallest $\sigma$-algebra containing the zero sets in $P$. By Theorem 1 in [4] every analytic metrizable space is a Lusin space, and trivially, every absolute Borel set is a Lusin space (every absolute Borel set is a Baire set in every metrizable space in which it is embedded). A similar problem can be posed by replacing the class of all metrizable spaces by the class of all completely regular spaces.

It would be useful to find an extent class of reasonable mappings preserving absolute Souslin sets in the class of metrizable spaces. Analytic spaces are preserved by continuous mappings; on the other hand, every metrizable space is a one-to-one continuous image of a discrete space. A rather small class is described in the next theorem.

THEOREM 5. Let $f: P \rightarrow Q$ be a proper surjective mapping of metrizable spaces. If one is an absolute Souslin space then so is the other one.

Proof. If $Q$ is an absolute Souslin space then, clearly, so is $P$ by Theorem 2, condition (b), because $f$ extends to a proper map $g$ of $\beta P$ onto $\beta Q$ and the preimage under a proper mapping of a $G_{i}$ (a Souslin set) if obviously a $G_{\delta}$ (a Souslin set). Conversely, assume $P=G \cap A$ where $G=\bigcap\left\{U_{n}\right\}, U_{n}$ open, and $A=\boldsymbol{S}(F), F(s)$ closed in $P$. Put $K_{n}=\beta P-U_{n}, \quad V_{n}=\beta Q-g\left[K_{n}\right], \quad E(s)=g[F(s)], \quad A^{\prime}=$ $\boldsymbol{S}(E), G^{\prime}=\bigcap\left\{V_{n}\right\}$. Clearly $G^{\prime}$ is a $G_{o}$ and $A^{\prime}$ is Souslin in $\beta Q$. Evidently, $A^{\prime} \cap G^{\prime} \supset Q$. If $x \in A^{\prime} \cap G^{\prime}$, then $g^{-1} x \subset G$ and $\mathrm{g}^{-1} x \cap A \neq$ $\varnothing$. If $y \in g^{-1} x \cap A$, then $y \in P$ because $G \cap A=P$, and so $x=g y \in Q$.

The next theorem of this section follows from Theorems 2 and 4 , and Theorems in [5].

THEOREM 6. A space $P$ is the intersection of $a G_{o}$ with a Souslin set derived from the zero sets of $\beta P$ if and only if there exists a continuous mapping $F$ of $\Sigma$ into $C(P)$ and a sequence $\left\{I_{n}\right\}$ of closed free ideals in $C(P)$ such that any maximal ideal $I$ is fixed if and only if I contains some $F(\sigma)$ but no $I_{n}$.

It is clear that absolute Souslin sets in a metrizable space are preserved by the Souslin operation. The next theorem states that the same is true in any space for Souslin sets satisfying the equivalent 
conditions in Theorem 2. Thus these sets seem to form a reasonable generalization of the absolute Souslin sets to nonmetrizable spaces, without any restriction of separability.

THeorem 7. Let $P$ be a space and let $\mathscr{A}$ be the collection of all Souslin sets $X$ in $P$ satisfying the equivalent conditions (a)-(d) in Theorem 2 with $X$ instead of $P$. Then $S(\mathscr{A})=\mathscr{A}$.

Proof. Take a compactification $K$ of $P$. Observe that $X \in \mathscr{A}$ if and only if $X=A_{X} \cap G_{X}$ with $A_{X}$ analytic (in $K$ ) and $G_{X}$ a $G_{\delta}$ in $K$, $P \subset G_{X}$. Now, if $M$ is a mapping of $S$ into $\mathscr{A}$, and if $F(s)$ is $A_{M(s)}$ for each $s$, then

$$
S(M)=G \cap S(F)
$$

where $G$ is the intersection of all $G_{M(s)}$. Thus $S(M) \in \mathscr{A}$ because $G$ is a $G_{r}$, and $\boldsymbol{S}(F)$ is analytic.

REMARK. The collection $\mathscr{A}$ in Theorem 7 need not contain all subspaces satisfying the equivalent conditions in Theorem 2.

The next lemma tells something about the relation to Souslin sets derived from the open sets.

Lemma 4. Let $K$ be a compactification of $P$, and let $F$ be a determining system of closed sets in $P$ such that $S(F)=P$. There exists a $G_{o}$ set $G$ in $K$ such that

$$
\text { (*) } \quad G \cap S\left(\operatorname{cl}_{K}[F]\right)=P
$$

if and only if there exists a determining system $U$ of open sets in $K$ such that $U(s) \supset F(s)$ for all $s$ in $S$, and

$$
S(U)=P \text {. }
$$

Proof. Assume (*), take open $U_{n}$ in $K$ with $\bigcap\left\{U_{n}\right\}=G$, and consider the collection $\mathscr{U}_{n}, n \in N$, of all open sets in $K$ with the closure contained in $U_{n}$. Let $U(s)$ be the star of $F(s)$ in $\mathscr{C}_{n}$ where $n$ is the length of $s$. It is routine to check the relation $S(U)=P$. Conversely, let $\mathscr{U}(s)$ be the collection of all open sets in $P$ whose closures (taken in $K$ ) are contained either in $U(s)$ or in the complement of $F(s)$. The struct $\langle\{\mathscr{Q}(s) \mid s \in S\}, F\rangle$ is complete, and Lemma 1 applies.

REMARK. It should be remarked that a similar result can be easily formulated without any reference to $K$, see Theorem 16 . 
2. Absolute Borel spaces. The condition in the next theorem is an analog of a characterization of Borelian spaces, see [2, Th. 7]. I don't know whether this condition is necessary (see Theorem 11). This result will not be used in the sequel.

THEOREM 8. The following condition is sufficient for a metrizable space $P$ to be an absolute Borel space: there exists a metric $d$ for $P$ and a proximal dusco correspondence of $\Sigma$ onto $\langle P, d\rangle$ such that the values are complete.

The phrase "proximal dusco" means that $f \sigma$ is distant to $f \tau$ for $\sigma \neq \tau$ (proximal disjointness $-d$ in dusco), and for every proximal neighborhood $U$ of any $f \sigma$ (i.e., $U$ contains a sphere about $f \sigma$ ) there exists a neighborhood $V$ of $\sigma$ with $f \tau \subset U$ for $\tau$ in $V$ (proximal usco).

Proof of Theorem 8. Let $\langle Q, D\rangle$ be a completion of $\langle P, d\rangle$. We shall prove that $P$ is a Borel set in $Q$. There exists a mapping $B$ of $S$ into the Borel sets in $Q$ such that each collection $\left\{B(s) \mid s \in S_{n}\right\}$ is disjoint and $B(s) \supset f[\Sigma(s)]$ for all $s$. Indeed, if it were not true then we would construct distinct $\sigma$ and $\tau$ in $\Sigma$ such that no pair $f\left[\Sigma\left(\sigma_{n}\right)\right], f\left[\Sigma\left(\tau_{n}\right)\right]$ would admit a "separation" by a Borel set in $Q$. Since $f$ is proximally dusco there exists an $n$ such that the above sets are distant in $P$, and so in $Q$, thence they are separated in $Q$. The remainder is clear. Define $F$ by setting $F(s)=B(s) \cap \operatorname{cl}_{Q} f[\Sigma(s)]$. Without any lost of generality we may and shall assume that $F$ is a determining system. It follows from the proximal upper semicontinuity of $f$ that

$$
S\left(\left\{\mathrm{cl}_{Q} f[\Sigma(s)]\right\}=P,\right.
$$

hence $S(F)=P$. The collections $\left\{F(s) \mid s \in S_{n}\right\}$ are disjoint, and hence

$$
P=S(F)=\bigcap\left\{\bigcup\left\{F(s) \mid s \in S_{n}\right\} \mid n \in N\right\} .
$$

The sets $F(s)$ are Borel sets in $Q$, and hence $P$ is a Borel set in $Q$.

Now the absolute Borel sets will be investigated by means of methods similar to those used in $\S 1$. For convenience we introduce a name for the structure corresponding to the determining system in $\S 1$.

Definition. A Borel structure on a space $P$ is defined to be a determining system $M: S \rightarrow \exp P$ such that each $\left\{M(s) \mid s \in S_{n}\right\}$ is a disjoint covering of $P$, and for every two distinct points $\sigma$ and $\tau$ in $\Sigma$ there exists an $n$ in $N$ such that

(*) the sets $M\left(\sigma_{n}\right)$ and $M\left(\tau_{n}\right)$ are functionally separated.

A Borel structure on a proximity space $P$ (in particular, on a uniform 
space or a metric space) is a Borel structure $M$ on the underlying topological space such that, for each distinct $\sigma$ and $\tau$ in $\Sigma$, there exists an $n$ such that

(**) the sets $M\left(\sigma_{n}\right)$ and $M\left(\tau_{n}\right)$ are distant.

Self-evidently $(* *)$ implies $(*)$. It is easy to see that a Borelian structure on a topological space $P$ (cf. [2, Definition 4, p. 163]) is essentially a Borel structure on any proximity space inducing the topology of $P$.

Recall that a Borelian structure on a space $P$ is a complete sequence $\left\{\mathscr{L}_{n}\right\}$ of countable disjoint coverings of $P$ such that $\mathscr{L}_{n+1}$ refines $\mathscr{L}_{n}$, and

$$
\bigcap\left\{\operatorname{cl} M_{n}\right\} \cap \bigcap\left\{\operatorname{cl} N_{n}\right\}=\varnothing
$$

whenever $M_{n}, N_{n} \in \mathscr{C}_{n}, M_{n} \supset M_{n+1}, N_{n} \supset N_{n+1}$, and $M_{k} \neq N_{k}$ for some k. It follows that $\bigcap\left\{\mathrm{cl} M_{n}\right\}=\bigcap\left\{M_{n}\right\}$ is a compact set and each neighborhood of this compact set contains cl $M_{n}$ for large enough $n$. Thence, given a proximity on $P, M_{n}$ and $N_{n}$ must be distant for large enough $n$. A Borelian structure is a sequence of coverings with some properties, and a Borel structure is a determining system with some properties, and hence no Borelian structure is a Borel structure. On the other hand, given a Borelian structure $\left\{\mathscr{M}_{n}\right\}$, it is easy to construct a determining system $M$ such that each element of $\left\{M s \mid s \in S_{n}\right\}$ either belongs to $\mathscr{C}_{n}$ of is empty. Now it is easy to prove that $M$ is a Borel structure on $P$, and this is the meaning of the sentence that a Borelian structure is essentially a Borel struture on any proximity space inducing the topology of $P$. In addition, obviously $M$ is a complete Borel structure on $P$. Conversely, if $M$ is a complete Borel structure on a space $P$, then $\left\{\left\{M s \mid s \in S_{n}\right\} \mid n \in N\right\}$ is a Borelian structure on $P$. In what follows by a Borelian structure on $P$ we shall mean a complete Borel structure on $P$. Before going on we must review a few results from [2] and [3].

Recall that a set with a disjoint Souslin representation in a space $P$, shortly a $d$-Souslin set in $P$, is a set of the form $S(F)$ with all $F s$ closed, and $\{F(\sigma) \mid \sigma \in \Sigma\}$ disjoint. Now, $P$ is Borelian if and only if $P$ is a $d$-Souslin set in some and then any compact space $Q \supset P$. If $\mathscr{M}$ is a collection of sets then $\boldsymbol{B}_{d}(\mathscr{C})$ denotes the smallest collection of sets containing $/ \mathscr{C}$ and closed under countable intersections and countable disjoint unions. Now, $P$ is Borelian if and only if $P \in \boldsymbol{B}_{d}$ (Lindelöf locally compact sets of $Q$ ) for some and then any compact space $Q \supset P$. It is easy to see that Lindelof locally compact sets in a compact space $Q$ are just the intersections of cozero sets in $Q$ with closed sets in $Q$. The elements of 


\section{$\boldsymbol{B}_{d}($ cozero sets in $Q)$}

are called Baire sets in $Q$. In metrizable, more generally in perfectly normal spaces, open sets and cozero sets coincide, and so any Borelian space is a Baire set in any metrizable space containing it. Moreover, every Borelian space is analytic, hence Lindelöf, and hence every metrizable Borelian space is a separable absolute Borel set.

REMARK. If $P$ belongs to $\boldsymbol{B}_{d}$ (closed set in $Q$ ) for some compact space $Q$ then $P$ is Borelian. On the other hand, the open interval $(0,1)$ does not belong to $\boldsymbol{B}_{d}$ (closed set in $\left.[0,1]\right)$ where $[0,1]$ denotes the closed interval.

THEOREM 9. The following condition (a)-(c) on a space $P$ are equivalent: $\beta P$;

(a) $P$ is the intersection of $a G_{o}$ with a Borelian subspace of

(b) $P$ is the intersection of a $G_{\delta}$ with a Borelian subspace in some tight extension $K$ of $P$.

(c) There exists a sequence $\left\{\mathscr{U}_{n}\right\}$ of open coverings of $P$ and $a$ Borel structure $M$ on $P$ such that $\left\langle\left\{\mathscr{U}_{n}\right\}, M\right\rangle$ is complete.

Proof. Evidently (a) implies (b), We derive (c) from (b). Let $P=G \cap B$ where $G$ is a $G_{\delta}$ and $B$ is a Borelian subspace in some tight extension $K$ of $P$. Choose open sets $U_{n}$ in $K$ with $G=\bigcap\left\{U_{n}\right\}$, and a Borelian structure $F$ on $B$. Put $M(s)=P \cap F(s)$ for $s$ in $S$, and for each natural $n$, let $\mathscr{U}_{n}$ denote the set of all open sets $V$ in $P$ with $\operatorname{cl}_{K} V \subset U_{n}$. Clearly $M$ is a Borel structure on $P$, and it is easy to show that $\left\langle\left\{\mathscr{U}_{n}\right\}, M\right\rangle$ is complete.

Finally, to prove that (c) implies (a), assume that $\left\langle\left\{\mathscr{Q}_{n}\right\}, M\right\rangle$ is complete. For each natural $n$ consider the union $U_{n}$ of all open sets $V$ in $K=\beta P$ with $V \cap P \in \mathscr{U}_{n}$, and put $G=\bigcap\left\{U_{n}\right\}$. Next, put $B=S(F)$ where $F=\{s \rightarrow F(s)\}$ is defined by the formula

$$
F(s)=\bigcup\left\{\bigcap\left\{\mathrm{cl}_{K} M(t) \mid t<\sigma\right\} \mid s<\sigma\right\} .
$$

It follows by the argument used to prove Lemma 1 that $P=G \cap B$. It remains to show that $B$ is a Borelian space, which we do by showing that $F$ is a complete Borel structure on $B$. Evidently $F$ is a determining system on $B$. The condition that $\left\{F(s) \mid s \in S_{n}\right\}$ are disjoint follows from the definition and the property $(*)$ that will be verified below. If $\mathscr{C}$ is a Cauchy filter on $\langle B, F\rangle$ then $F(s) \in \mathscr{C}$ for all $s<\sigma$, where $\sigma$ is a certain element of $\Sigma$, and hence every cluster point of $\mathscr{C}$ in $K$ is contained in each $\operatorname{cl}_{K}(s), s<\sigma$, and so in $B$ which proves that $\mathscr{C}$ has a cluster point in $B$. To prove 
the property $(*)$ (for $F$ ) in the definition of Borel structure, consider two distinct elements $\sigma$ and $\tau$ of $\Sigma$; for some natural $n$ the sets $M\left(\sigma_{n}\right)$ and $M\left(\tau_{n}\right)$ are functionally separated in $P$, and hence in $\beta P$. Thus $F\left(\sigma_{n}\right)\left(\subset \operatorname{cl} M\left(\sigma_{n}\right)\right)$ and $F\left(\tau_{n}\right)$ are functionally separated in $\beta P$, and hence in $B$. The proof is complete.

THEOREM 10. The following two conditions $(\mathrm{d})$ and $\left(\mathrm{d}^{\prime}\right)$ are equivalent for any space $P$, and imply conditions (a)-(c) in Theorem 9. If $P$ is paracompact then the conditions (a), (b), (c), (d), and $\left(d^{\prime}\right)$ are equivalent to each other.

(d) There exists a Borel structure $M$ on $P$ and a continuous pseudometric $d$ on $P$ such that $\langle d, M\rangle$ is complete on $P$.

$\left(d^{\prime}\right)$ Condition (d) holds, and whenever $\langle d, M\rangle$ is complete for some Borel structure $M$ on $P$ and a continuous pseudometric $d$ on $P$, then there exists a continuous pseudometric $D$ on $P$ such that $\langle D, M\rangle$ is complete and $M$ is a Borel structure on $\langle P, D\rangle$.

Proof. Condition (d) implies (c) for any $P$ because a filter $\mathscr{C}$ on $P$ is $d$-Cauchy if and only if it is a Cauchy filter with respect to $\left\{\mathscr{U}_{n}\right\}$ where $\mathscr{U}_{n}$ is the $1 / n$-covering of $P$ (here the $r$-covering of $\langle P, d\rangle$ is the collection of all open $r$-spheres in $\langle P, d\rangle$ ). If (c) holds, then $\langle d, M\rangle$ is complete for any continuous pseudometric $d$ on $P$ such that each $\mathscr{U}_{n}$ is refined by some $r$-covering of $\langle P, d\rangle$; such a $d$ exists if $P$ is paracompact. It remains to show that $(d)$ and $\left(d^{\prime}\right)$ are equivalent for any space $P$. Trivially (d') implies (d). Assuming (d), the required $D$ is of the form $d+\rho$ where $\rho$ is described in the next lemma.

LemMA 5. If $M$ is a Borel structure on a space $P$ then there exists a continuous pseudometric $\rho$ on $P$ such that $M$ is a Borel structure on $\langle P, \rho\rangle$.

Proof. If $s, t \in S_{n}, n \in N$, choose a continuous nonnegative function $f_{s, t} \leqq 1$ which is zero on $M(s)$ and one on $M(t)$, whenever such a function exists at all. Arrange all $f_{s, t}$ in a sequence $\left\{f_{n}\right\}$ and put $\rho\langle x, y\rangle=\Sigma\left\{\left|f_{n} x-f_{n} y\right| / 2^{n}\right\}$. It follows from $(*)$ in the definition of Borel structure that $M$ is Borel on $\langle P, \rho\rangle$.

Lemma 6. Let $\langle Q, D\rangle$ be a completion of a metric space $\langle P, d\rangle$. Then $P$ is a Borel set in $Q$ if and only if there exists a Borel structure $M$ on $\langle P, d\rangle$ such that $\langle d, M\rangle$ is complete.

Proof. Assume that $P$ is a Borel set in $Q$, and hence a Baire set in $Q$. Consider the compactification $K$ of $Q$ corresponding to the 
proximity structure induced by $D$. Clearly every Baire set in $Q$ is the intersection of $Q$ with a Baire set in $K$. Hence $P=Q \cap B$ where $B$ is a Baire set in $K$. By the results stated before Theorem $9, B$ is Borelian; let $F$ be a Borelian structure on $B$. Then $F$ is also a Borel structure on the proximity subspace $Q$ of $K$, and hence the structure $M$ induced by $F$ on $Q \cap B=P$ is a Borel structure on the metric space $P$. One easily checks that $\langle d, M\rangle$ is complete.

Conversely, assuming that $\langle d, M\rangle$ is complete where $M$ is a Borel structure on $\langle P, d\rangle$, we shall prove that $P$ is a Borel set in $Q$. Observe that there exists a determining system $Z$ of Baire sets in $Q$ such that each collection $\left\{Z(s) \mid s \in S_{n}\right\}$ is disjoint, and $Z(s) \supset M(s)$ for all $s$. Indeed, it is enough to show that for each distinct $s$ and $t$ in $S_{n}, n=1,2, \cdots$, there exists a Baire set $B$ in $Q$ such that $M(s) \subset B$, $B \cap M(t)=\varnothing \quad$ (say that $B$ separates $M(s)$ and $M(t))$. If some $M(s)$ and $M(t)$ were not separated, then there would exist (distinct) $\sigma$ and $\tau$ in $\Sigma$ such that $s=\sigma_{n}, t=\tau_{n}$, and no pair $M\left(\sigma_{m}\right), M\left(\tau_{m}\right)$ would admit a separation. But this would contradict $(* *)$ in the definition of Borel structures. To conclude the proof put

$$
B(s)=\left(\mathrm{cl}_{Q} M(s)\right) \cap Z(s) .
$$

Evidently $B(s)$ is a Baire set in $Q$, and $S(B)=P$ because $S\left(\operatorname{cl}_{Q}[M]\right)=$ $P$. Since each $\left\{B(s) \mid s \in S_{n}\right\}$ is disjoint, we get

$$
S(B)=\bigcap\left\{\bigcup\left\{B(s) \mid s \in S_{n}\right\} \mid n \in N\right\}
$$

which proves that $S(B)$ is a Baire set in $Q$, and concludes the proof.

Lemma 7. Assume that $P$ is metrizable and $\langle d, M\rangle$ is complete for some metric $d$ of $P$, and some Borel structure $M$ on $\langle P, d\rangle$. Let $D$ be any metric for $P$. Then there exists a Borel structure $F$ for $\langle P, D\rangle$ such that $\langle D, F\rangle$ is complete.

Proof. ${ }^{2}$ This is an immediate consequence of Lemma 7 and the fact that a Borel set in a completely metrizable space is absolutely Borel.

Theorem 11. If $P$ is metrizable then each of the conditions (a) through $\left(\mathrm{d}^{\prime}\right)$ in Theorems 9 and 10, and also each of the conditions (e) through (h) listed below, is necessary and sufficient for $P$ to be an absolute Borel set.

(e) If $d$ is a metric for $P$ then there exists a Borel structure

2 One can prove Lemma 7 independently of Lemma 6 . The direct proof is interesting and instructive, but too long. The author intends to publish it elsewhere in connection with a deeper investigation of completions of metric spaces. 
$M$ on $\langle P, d\rangle$ such that $\langle d, M\rangle$ is complete.

(f) If $d$ is a metric for $P$, and $\langle R, D\rangle$ is a completion of $\langle P, d\rangle$, then there exists a Baire set $B$ in the compactification of $R$ corresponding to the proximity induced by $D$, such that $B \cap R=P$.

$(\mathrm{g})$ For every completion $R$ of $P$ there exists a Baire set $B$ in $\beta R$ such that $B \cap R=P$.

(h) $P$ is the intersection of $a G_{o}$ with a Baire set in $\beta P$.

Proof. Evidently (g) is necessary and sufficient, and (h) implies (a). Condition ( $d^{\prime}$ ) implies (e) by Lemma 7, and obviously (e) implies (f) by Lemma 6 . The implications (f) $\Rightarrow(\mathrm{g}) \Rightarrow$ (h) follow by considering the canonical mapping of $\beta R$ onto $K$, and of $\beta P$ onto $\beta R$, respectively.

REMARK. An analog of Theorem 8 does not hold. We shall return to this question in $\S 3$ where another generalization of absolute Borel sets will be introduced.

The section is concluded by a formal but important, restatements of conditions in Theorems 2, 3, 4, 9, and 11 .

Theorem 12. (a) A space $P$ is an absolute Borel (Souslin) space if and only if $P$ is a closed subspace of the product $R \times B$, where $R$ is a completely metrizable space (which may be taken to be a completion of $P$ ) and $B$ is a separable absolute Borel (Souslin) space.

(b) A space $P$ is the intersection of a $G_{\delta}$ set with an analytic (Borelian) subspace in $\beta P$ if and only if $P$ is a closed subspace of a product space $R \times B$ such that $R$ is a topologically complete space in the sense of $E$. Čech and $B$ is an analytic (Borelian) space.

REMARK. Separable absolute Souslin spaces coincide with metrizable analytic spaces, and separable absolute Borel spaces coincide with metrizable Borelian (equivalently, bianalytic) spaces.

Proof of Theorem 12. "If" is clear. For "only if" consider first the simpler case (b). Assume that $P=R \cap B$, where $R$ is a $G_{o}$ in $\beta P$, and $B$ is analytic (Borelian) in $\beta P$. Since $\beta P$ is separated, the diagonal $P^{\prime}$ of $R \times B$ is closed in $R \times B$, and clearly $P^{\prime}$ is homeomorphic to $P$. To prove "only if" in (a), assume that $P$ is absolutely Souslin (Borel). By Theorem 4 (Theorem 11, condition (g)) $P=R \cap B$ where $R$ is a completion of $P$, and $B$ is a Souslin set derived from the zero sets (a Baire set) in $\beta R$. Take a continuous mapping $f$ of $\beta R$ onto a metrizable space such that $f[B] \cap f[\beta R-B]=\varnothing$; the 
existence of $f$ for $B$ a Baire set is given by a Halmos Theorem, and the proof for $B$ a Souslin set follows the same idea. The mapping $f$ is proper, and hence the product $h$ of the identity mapping of $R$ and of the restriction of $f$ to a mapping of $B$ onto $f[B]$ is also proper, and hence the restriction $h \mid P^{\prime}$ of $h$ to the diagonal $P^{\prime}$ of $R \times B$ (which is closed in $R \times B$ ) is also proper. Since $h \mid P^{\prime}$ is one-to-one, it is a homeomorphism. Thus the closed subspace $h\left[P^{\prime}\right]$ of $R \times f[B]$ is a homeomorph of $P$.

For the next theorem we need the following simple consequence of the Lavrentiev Theorem.

Proposition. Let $f$ be a continuous mapping of a subset $X$ of a metrizable space $P$ into an absolute Borel space $Q$. Then $f$ extends to a continuous mapping of a Borel set $B \supset X$ in $P$ into $Q$.

Proof. Let $R$ be a completion of $Q$. By the Lavrentiev Theorem $f$ extends to a continuous mapping $F$ of a $G_{\delta}$ set $G \supset X$ in $P$ into $R$. Clearly the restriction of $F$ to $f^{-1}[Q]$ is the required extension.

THEOREM 13. A metrizable space $P$ is absolutely Borel if and only if for some (and then any) completion $R$ of $P$ there exists a continuous mapping of $P$ into a separable absolute Borel space $B$ which is nonextensible to any point of $R-P$.

Proof. Assume that a continuous $f: P \rightarrow B$ is nonextensible to any point of a completion $R$ of $P$, where $B$ is a separable absolute Borel set. By Proposition the mapping $f$ extends to a continuous mapping of a Borel set $C \supset P$ in $R$ into $B$. By our assumption $C=$ $P$, and hence $P$ is a Borel set in $R$, hence an absolute Borel set. Conversely, assume that $P$ is an absolute Borel space, and let $R$ be a completion of $P$. There exists a Baire set $B$ in $\beta R$ such that $B \cap R=P$. Take a continuous mapping $g$ of $\beta R$ onto a compact metrizable space $Q$ such that $B=g^{-1}\left[B^{\prime}\right]$. Let $f$ be the restriction of $g$ to a mapping of $P$ into $B^{\prime}$. The space $B^{\prime}$ is a separable absolute Borel space, and $f$ is nonextensible to any point of $R-P$.

It should be remarked that obvious analogs of Theorems 12 and 13 hold for absolute distinguishable sets, and Theorem 13 holds for absolute Souslin sets. Recall that a set $X$ in a space $P$ is said to be distinguishable if there exists a continuous mapping $f$ of $P$ into a separable metrizable space such that $f[X] \cap f[P-X]=\varnothing$. The proofs go over. 
Finally, in Theorem 13 one can replace "continuous" by "uniformly continuous" with obvious additional changes.

The last result is a restatement of the condition related to $C(P)$. See [5, Th. 1].

THEOREM 14. A space $P$ is the intersection of a $G_{\delta}$ with a Baire set in $\beta P$ if and only if there exists a decreasing sequence $\left\{I_{n}\right\}$ of closed free ideals in $C(P)$, and a continuous mapping $F$ of $\Sigma$ into $C(P)$ such that:

(a) No maximal ideal contains the intersection of two ideals generated by the values of $F$ at two distinct points of $\Sigma$, and

(b) A maximal ideal is fixed if and only if it contains the ideal generated by some $F \sigma, \sigma \in \Sigma$.

Corresponding characterizations of $P^{\prime} \mathrm{s}$ which are intersections of a $G_{\delta}$ with respectively a $Z$-Souslin or absolutely distinguishable set are simpler because the rather complicated condition (a) is not needed.

3. Absolute $U$-Borel spaces. Here another generalization of absolute Borel sets will be treated. If $\mathscr{C l}$ is a collection of sets let $\boldsymbol{B}(\mathscr{C})$ denote the smallest collection containing $\mathscr{C}$ and closed under countable intersections and countable unions. Thus $\boldsymbol{B}(\mathscr{C}) \supset \boldsymbol{B}_{d}(\mathscr{C})$, and it is a classical result that the two collections coincide if $\mathscr{C}$ is the collection of all cozero-sets in a space.

Definition. A set $X$ in a space $P$ is called $U$-Borel ( $F$-Borel) if $X$ belongs to $B(\mathscr{K})$ where $\mathscr{C}$ is the collection of all open (closed) sets in $P$. A set $X$ in $P$ is called $U$-Souslin if $X$ is a Souslin set derived from the open sets in $P$.

In any perfectly normal space, in particular in any metrizable space, every closed set, and also every open set in a Baire set; in consequence, Baire sets, $U$-Borel sets and $F$-Borel sets coincide. Also the $Z$-Souslin, Souslin and $U$-Souslin sets coincide in such a space. By a $Z$-Souslin set in a space we mean a Souslin set derived from the zero sets in the space. In any space $P$ a set $X$ is $U$-Borel if and only if $P-X$ is $F$-Borel, but a corresponding statement for "Souslin" is not true.

It is evident that if $f$ is a continuous mapping of $P$ into $Q$, and $X \subset Q$ is $F$-Borel, $U$-Borel, Souslin or $U$-Souslin in $Q$, then $f^{-1}[X]$ has the respective property in $P$. 
THEOREM 15. ${ }^{3}$ The following conditions on a space $P$ are equivalent:

(i) $P$ is $U$-Borel in $\beta P$.

(j) $P$ is $U$-Borel in some compactification of $P$.

(k) $P$ is $U$-Borel in every compactification of $P$.

(1) $P$ is $U$-Borel in every tight extension of $P$.

Further, the same holds with $U$-Borel replaced by U-Souslin throughout.

Definition. A space satisfying the equivalent conditions (i) through (l) will be called an absolute $U$-Borel space. If we replace $U$-Borel by $U$-Souslin we get the definition of absolute $U$-Souslin spaces.

COROLlary. Each of the conditions (i) through (l) is necessary and sufficient for a metrizable space to be an absolute Borel (absolute Souslin) space.

Proof of Theorem 15 for U-Souslin sets. It is enough to prove: if $K$ and $L$ are compactifications of $P$, and if there exists a canonical mapping $f$ of $K$ onto $L$, and if $P$ is $U$-Souslin in $K$, then $P$ is $U$ Souslin in $L$. Assume $P=\boldsymbol{S}(U)$ where $U$ consists of open sets in $K$; define $V$ by setting $V(s)=E\left\{y \mid y \in L, f^{-1} y \subset U(s)\right\}$. Clearly $V$ consists of open sets in $L$ and $\boldsymbol{S}(V)=P$. For a simple proof for $U$-Borel sets we need the following lemma which is of some interest in itself.

LeMma 8. $A$ set $X$ in a space $P$ is U-Borel (equivalently $P-X$ is $F$-Borel) if and only if there exist determining systems $U$ and $F, U$ consists of open sets and $F$ consists of closed sets, such that $\boldsymbol{S}(U)=X, \boldsymbol{S}(F)=P-X$, and for each $\sigma, \tau \in \Sigma$

$$
U\left(\sigma_{n}\right) \cap F\left(\tau_{n}\right)=\varnothing \text { for some } n \text {. }
$$

Proof. To prove "only if" it is enough to show that the sets $X$ satisfying the condition are preserved by countable unions and countable intersections. We omit the routine details. To prove "if", consider the determining systems $L$ and $K$ defined by setting

$$
\begin{aligned}
& L(s)=\bigcup\{U(\sigma) \mid s<\sigma\}, \\
& K(s)=\bigcup\{F(\sigma) \mid s<\sigma\} .
\end{aligned}
$$

3 The part concerning $U$-Borel sets has been essentially published by S. Willard, Absolute Borel sets in their Stone-ČCech compactifications, Fund. Math. 58 (1966), 323-333. I am grateful for this reference to the referee and A. H. Stone. 
Clearly $L(s) \subset U(s), K(s) \subset F(s), \boldsymbol{S}(L)=X, \boldsymbol{S}(K)=P-X$, and

$$
\begin{aligned}
& L(s)=U\{L(\{s, i\}) \mid i \in N\} \\
& K(s)=U\{K(\{s, i\}) \mid i \in N\},
\end{aligned}
$$

where $\{s, i\}$ stands for the sequence $\left\{s_{0}, \cdots, s_{n}, i\right\}$ with $s=\left\{s_{k} \mid k \leqq n\right\}$. If $X$ were not a $U$-Borel set, i.e., if $X \subset Y \subset P-(P-X)$ for no $U$-Borel set $Y$, then, because of $(* *)$, there would exist indices $i_{0}$ and $j_{0}$ such that

$$
L\left(\left\{i_{0}\right\}\right) \subset Y \subset P-K\left(\left\{j_{0}\right\}\right)
$$

for no $U$-Borel set $Y$. Proceeding by induction, there would exist $\sigma$ and $\tau$ in $\Sigma$ such that

$$
L\left(\sigma_{n}\right) \subset Y \subset P-K\left(\tau_{n}\right)
$$

for no $U$-Borel set $Y$. However, this would contradict condition $(*)$.

Proof of Theorem 15 for $U$-Borel. Assume $P$ is $F$-Borel in a compactification $K$ of $P$, and let $f$ be the natural mapping onto a compactification $L$ of $P$. By Lemma 8

$$
P=S(U), K-P=S(F)
$$

where $U(s)$ is open, and $F(s)$ is closed for each $s$ in $S$, and condition $(*)$ is fulfilled. Define $U^{\prime}$ and $F^{\prime}$ by setting

$$
U^{\prime}(s)=E\left\{x \mid f^{-1} x \subset U(s)\right\}, F^{\prime}(s)=f[F(s)] .
$$

Lemma 8 applies.

It should be remarked that Lemma 8 is the main tool for proving (B) and (C) in Theorem 16 . Theorem 15 can be proved elementary just by considering the mapping that assigns to each open set $U$ in $K$ the open set $E\left\{x \mid f^{-1} x \subset U\right\}$ in $L$.

REMARK. In Lemma 8 it is not important that we are working in a topological space with closed and open sets. Let $P$ be a set, $\mathscr{F}$ a collection of subsets of $P$, and let $\mathscr{Q}$ consist of the complements of sets in $\mathscr{F}$. Then $X \in \boldsymbol{B}(\mathscr{F})$ if and only if there exist mappings $F: S \rightarrow \mathscr{F}$ and $U: S \rightarrow \mathscr{C}$ such that $X=S(F), P-X=$ $S(U)$, and for each $\sigma, \tau \in \Sigma$ there exists an $n$ with $F\left(\sigma_{n}\right) \cap U\left(\tau_{n}\right)=\varnothing$.

I know nothing about an analog of Theorem 15 for $F$-Borel sets. Anyway, it is clear that every space $P$ which is $F$-Borel in $\beta P$ is analytic, hence Lindelöff, and hence separable if metrizable. So no direct description of nonseparable absolute Borel sets can be given in 
this way. However, we would get a very good generalization of absolute Borel sets if "absolute $F$-Borel" sets were able to play the role of Borelian spaces in $\S 2$. This is shown by the following observation:

Let $K$ be a tight extension of a space $P$, and let $\mathscr{l l}$ be the collection of all sets $X$ in $P$ such that $X=G \cap Y$ for some $G_{o}$ set $G$ in $K, G \supset P$, and some $F$-Borel set $Y$ in $K$. Then $B(\mathscr{C}) \subset \mathscr{l}$.

In conclusion we give some internal characterizations of absolute $U$-Borel ( $U$-Souslin) spaces, and spaces $P$ which are $F$-Borel in $\beta P$.

For convenience, define a co-determining system to be a singlevalued relation with domain $S$ and the range contained in the class of all sets, such that $M(t) \supset M(s)$ whenever $s$ is a section of $t$. The operation co $S$, called the co-Souslin operation, assigns to each codetermining system $M$ the set

$$
\cos (M)=\bigcap\{\{\bigcup M(s) \mid s<\sigma\} \mid \sigma \in \Sigma\} .
$$

A co-determining system $M$ is on $P$, if $M(s) \subset P$ for all $s$ in $S$ and co $S(M)=P$. Finally, a co-determining system $M$ on a space $P$ is called complete if

$$
\{\{M(s) \mid s<\sigma\} \mid \sigma \in \Sigma\}
$$

is a complete family of coverings. The characterizations now read as follows.

Theorem 16. (A) A space $P$ is absolute U-Souslin if and only if there exists an open determining system $U$ on $P$ and a family $\{\mathscr{C}(s)\}$, $\mathscr{C}(s)$ being an open cover of $U(s)$ for each $s \in S$, such that if $\mathscr{C l}$ is a filter on $P$ with the property that there exists $\sigma$ in $\Sigma$ with $\mathscr{Q}(s) \cap \mathscr{l l} \neq \varnothing$ for all $s<\sigma$, then $\mathscr{C l}$ has a cluster point in $P$.

(B) A space $P$ is absolute U-Borel if and only if there exists $U$ and $\{\mathscr{C}(s)\}$ with the properties in $A$, and a complete co-determining system $N$ on $P$ consisting of open sets such that for each $\sigma$ and $\tau$ in $\Sigma$ there exists an $n$ with $U\left(\sigma_{n}\right) \subset N\left(\tau_{n}\right)$.

(C) A space $P$ is F-Borel in $\beta P$ if and only if there exist a complete determining system $L$ on $P$ (which may be assumed to consist of closed sets), and a complete co-determining system $E$ on $P$ consisting of closed sets such that for each $\sigma$ and $\tau$ in $\Sigma$ there exists an $n$ with $F\left(\sigma_{n}\right) \subset E\left(\tau_{n}\right)$.

Proof of (A). Firstly assume that $P$ is $U$-Souslin in a space $K$; thus $P=S(V)$ with all the $V(s)$ open in $K$. Define $U$ by setting $U(s)=P \cap V(s)$, and let $\mathscr{U}(s)$ consist of all open sets $G$ in $P$ such 
that the closure of $G$ in $K$ is contained in $V(s)$. If $\mathscr{C}$ is a filter on $P$, and if $G(s) \in \mathscr{M} \cap \mathscr{U}(s)$ for all $s<\sigma$ with some $\sigma$ in $\Sigma$, then each cluster point of $\mathscr{C}$ in $K$ must be contained in the intersection of $\{V s \mid s<\sigma\}$, and hence in $P$. Thus if $K$ is compact, then $\mathscr{M}$ has a cluster point in $P$. This proves "only if". Conversely, assume that $U$ and $\{\mathscr{C}(s)\}$ with the properties in $A$ are given, and let $K$ be any tight extension of $P$. For each open set $G$ in $P$ choose an open set $G^{\prime}$ in $K$ with $G^{\prime} \cap P=G$, and define a $V$ by setting

$$
V(s)=\bigcup\left\{G^{\prime} \mid G \in \mathscr{U}(s)\right\} \text {. }
$$

Clearly all $V(s)$ are open in $K$, and $\boldsymbol{S}(V) \supset P$. If $x \in \boldsymbol{S}(V) \backslash P$, then $x \in V(\sigma)$ for some $\sigma$ in $\Sigma$, and we can choose a family $\{G(s) \mid s<\sigma\}$ such that $G(s) \in \mathscr{U}(s)$ and $x \in(G(s))^{\prime}$ for all $s<\sigma$. Consider the neighborhood system $\mathscr{V}$ of $x$ in $K$, and let $\mathscr{C}$ consist of all $V \cap P$, $V \in \mathscr{Y}$. Since $K$ is separated, $x$ is the unique cluster point of $\mathscr{V}$, and hence of $\mathscr{M}$, in $K$. On the other hand, $G(s) \in \mathscr{M} \cap \mathscr{U}(s)$ for all $s<\sigma$. This contradicts the property of $\{\mathscr{U}(s) \mid s \cup S\}$ in $A$, and proves "if" in A.

Proof of (B). Assume that $P$ is $U$-Borel in a space $K$; by Lemma $8 P=S(V)$, and $K-P=S(F)$ such that all $U(s)$ are open in $K$, all $F(s)$ are closed in $K$, and for each $\sigma, \tau \in \Sigma$ there exists an $n$ with $V\left(\sigma_{n}\right) \cap F\left(\tau_{n}\right)=\varnothing$. Define $U$ and $\{\mathscr{U}(s) \mid s \in S\}$ as in the proof of "only if" in $A$. Define $H(s)=P \backslash F(s)$. The last property of $U$ and $H$ in $B$ is obviously fulfilled, and if $K$ is compact then all the properties are fulfilled by the proof of "only if" in $A$. Conversely, assume that $U,\{\mathscr{U}(s)\}$, and $H$ with the properties in $B$ are given, and let $K$ be a tight extension of $P$. Define $V$ as in the proof of "if" in $A$, and define $F$ by setting $F(s)$ to be the complement in $K$ of the largest open set $G(s)$ in $L$ with $G(s) \cap P=H(s)$. By the proof of $A$ we have $S(V)=P$, it follows from the completeness of $H$ that $S(F)=P$, and it is easy to check property (*) in Lemma 8 . By Lemma $8 P$ is $U$-Borel in $K$. This proves "if" in $B$.

Proof of (C). Assume that $P$ is $F$-Borel in a compactification $K$ of $P$. By Lemma 8 we can write

$$
P=S(F), \quad K-P=S(U)
$$

with $F(s)$ closed and $U(s)$ open in $K$ such that property $(*)$ is fulfilled. Define $L$ by setting $L(s)=F(s) \cap P, H$ by setting $H(s)=P-U(s)$. One can easily check all the properties required in $C$. Conversely, assume that $L$ and $H$ with the properties in $C$ are given, and let $K$ be a compactification of $P$. We shall apply Lemma 8 to prove that 
$P$ is $F$-Borel in $K$. For each $s$ in $S$ let $F(s)$ be the closure in $K$ of $L(s)$, and let $U(s)$ be the complement in $K$ of the closure $M(s)$ of $H(s)$ in $K$. The sets $F(s)$ are closed, the sets $U(s)$ are open, and it is easy to verify property $(*)$ in Lemma 8 . To apply Lemma 8 we need to prove that $S(F)=P$, and $S(U)=K-P$. The first relation follows from Lemma 1 (with $G=K$ ), the second need not hold in general. This is the place where we must assume that $K$ is a ČechStone compactification of $P$. The relation $S(U)=K-P$ is equivalent to the relation $\cos (M)=P$, and the latter relation holds by the following important result (Theorem 6 in [3]).

Lemma 9. Let $\left\{\mathscr{F}_{a} \mid a \in A\right\}$ be a complete family of closed coverings of a normal space $P$, and let $K$ be a Čech-Stone compactification of P. Then

$$
\bigcap\left\{\bigcup\left(\mathrm{cl}_{K} F \mid F \in \mathscr{F}_{a}\right\} \mid a \in A\right\}=P \text {. }
$$

It should be pointed out that for the proof the following property of Čech-Stone compactifications $K$ of normal spaces $P$ is needed: if $F_{1}$ and $F_{2}$ are closed in $P$ and $x \in K-P$ is in the closure of $F_{i}, i=1,2$, then $x$ is in the closure of $F_{1} \cap F_{2}$.

REMARK. Observe that the proof of Theorem 16 contains a new proof of Theorem 15.

The author is grateful to the referee for careful (and certainly tiring) work which made this paper partially readable.

\section{BIBLIOGRAPHY}

1. E. Čech, Topological spaces, Academia, Prague 1966.

2. Z. Frolík, "A contribution to the descriptive theory of sets and spaces, "General topology and its relations to modern analysis and algebra (Proc. Symp. Prague, Sept. 1961), Academic Press.

3. - On the descriptive theory of sets, Czech. Math. J. 13 (1963), 335-359.

4. - Baire sets that are Borelian subspaces, Proc. Roy. Soc. (A) 292 (1967),

5. - $-A$ note on $C(P)$ and Baire sets in compact and metrizable spaces, Bull. Acad. Polon. 15 (1967), 779-784.

6. - Structures in descriptive theory, Proceedings of the Topological Conference in Tempe, Arizona, 1967, 83-99.

7. J. D. Knowles and C. A. Rogers, Descriptive sets, Proc. Roy. Soc. (A) 291 (1965), 353-367.

8. K. Kuratowski, Topologie I, Warszawa, 1952.

9. D. Montgomery, Non-separable metric spaces, Fund. Math. 25 (1935), 527-534. 
10. A. H. Stone, Absolute $F_{\sigma}$-spaces, Proc. Amer. Math. Soc. 13 (1962), 495-499.

11. - Nonseparable Borel sets, Rozprawy Mat. 28, Warszawa, 1962.

Received June 1, 1967.

Louisiana State University

BATON ROUGE, LOUISIANA

Mathematics InStitute

CSAV, Praha 



\title{
PACIFIC JOURNAL OF MATHEMATICS
}

\author{
EDITORS
}

H. SAMELSON

Stanford University

Stanford, California 94305

J. DugunduI

Department of Mathematics

University of Southern California

Los Angeles, California 90007

RICHARD PIERCE

University of Washington

BASIL GORDON*

University of California

Seattle, Washington 98105

Los Angeles, California 90024

\section{ASSOCIATE EDITORS}

E. F. BeCKenbaCh

B. H. NeumanN

F. WOLF

K. YosHIDA

\section{SUPPORTING INSTITUTIONS}

\author{
UNIVERSITY OF BRITISH COLUMBIA \\ CALIFORNIA INSTITUTE OF TECHNOLOGY \\ UNIVERSITY OF CALIFORNIA \\ MONTANA STATE UNIVERSITY \\ UNIVERSITY OF NEVADA \\ NEW MEXICO STATE UNIVERSITY \\ OREGON STATE UNIVERSITY \\ UNIVERSITY OF OREGON \\ OSAKA UNIVERSITY \\ UNIVERSITY OF SOUTHERN CALIFORNIA
}

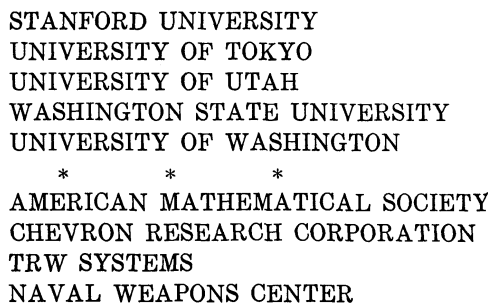

The Supporting Institutions listed above contribute to the cost of publication of this Journal, but they are not owners or publishers and have no responsibility for its content or policies.

Mathematical papers intended for publication in the Pacific Journal of Mathematics should be in typed form or offset-reproduced, double spaced with large margins. Underline Greek letters in red, German in green, and script in blue. The first paragraph or two must be capable of being used separately as a synopsis of the entire paper. It should not contain references to the bibliography. Manuscripts, in duplicate if possible, may be sent to any one of the four editors. Please classify according to the scheme of Math. Rev. 36, 1539-1546. All other communications to the editors should be addressed to the managing editor, Richard Arens, University of California, Los Angeles, California, 90024.

50 reprints are provided free for each article; additional copies may be obtained at cost in multiples of 50 .

The Pacific Journal of Mathematics is published monthly. Effective with Volume 16 the price per volume (3 numbers) is $\$ 8.00$; single issues, $\$ 3.00$. Special price for current issues to individual faculty members of supporting institutions and to individual members of the American Mathematical Society: $\$ 4.00$ per volume; single issues $\$ 1.50$. Back numbers are available.

Subscriptions, orders for back numbers, and changes of address should be sent to Pacific Journal of Mathematics, 103 Highland Boulevard, Berkeley, California, 94708.

PUBLISHED BY PACIFIC JOURNAL OF MATHEMATICS, A NON-PROFIT CORPORATION

Printed at Kokusai Bunken Insatsusha (International Academic Printing Co., Ltd.), 7-17, Fujimi 2-chome, Chiyoda-ku, Tokyo, Japan.

* Acting Managing Editor. 


\section{Pacific Journal of Mathematics}

\section{Vol. 32, No. 3 \\ March, 1970}

Shair Ahmad, Dynamical systems of characteristic $0^{+} \ldots \ldots \ldots \ldots \ldots$

Charles A. Akemann and Bernard Russo, Geometry of the unit sphere of a $C^{*}$-algebra and its dual............................ 575

Philip Bacon, The compactness of countably compact spaces ......... 587

Richard Blaine Barrar and Henry Loeb, On the continuity of the nonlinear Tschebyscheff operator ............................ 593

L. Carlitz, Factorization of a special polynomial over a finite field ....... 603

Joe Ebeling Cude, Compact integral domains .................... 615

Frank Rimi DeMeyer, On automorphisms of separable algebras. II . . . . . 621

James B. Derr, Generalized Sylow tower groups .................. 633

Raouf Doss, Some inclusions in multipliers ................... 643

Mary Rodriguez Embry, The numerical range of an operator........... 647

John Froese, Domain-perturbed problems for ordinary linear differential

operators..................................... 651

Zdeněk Frolík, Absolute Borel and Souslin sets ..................... 663

Ronald Owen Fulp, Tensor and torsion products of semigroups .......... 685

George Grätzer and J. Płonka, On the number of polynomials of an

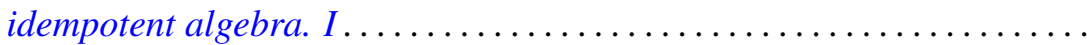

Newcomb Greenleaf and Walter Read, Positive holomorphic differentials on

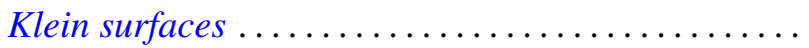

John Willard Heidel, Uniqueness, continuation, and nonoscillation for a second order nonlinear differential equation ................. 715

Leon A. Henkin, Extending Boolean operations................... 723

R. Hirshon, On hopfian groups .......................... 753

Melvin Hochster, Totally integrally closed rings and extremal spaces ..... 767

R. Mohanty and B. K. Ray, On the convergence of a trigonometric integral ..................................

Michael Rich, On a class of nodal algebras .................... 787

Emile B. Roth, Conjugate space representations of Banach spaces ........ 793

Rolf Schneider, On the projections of a convex polytope .............. 799

Bertram Manuel Schreiber, On the coset ring and strong Ditkin sets ...... 805

Edgar Lee Stout, Some remarks on varieties in polydiscs and bounded

holomorphic functions .........................

James Edward Ward, Two-groups and Jordan algebras . 